\title{
Scanning Quantum Dot Microscopy
}

\author{
Christian Wagner, ${ }^{1,2}$ Matthew F. B. Green, ${ }^{1,2}$ Philipp Leinen, ${ }^{1,2}$ Thorsten Deilmann, ${ }^{3}$ Peter Krüger, ${ }^{3}$ \\ Michael Rohlfing, ${ }^{3}$ Ruslan Temirov, ${ }^{1,2, *}$ and F. Stefan Tautz ${ }^{1,2}$ \\ ${ }^{1}$ Peter Grünberg Institut (PGI-3), Forschungszentrum Jülich, 52425 Jülich, Germany \\ ${ }^{2} J$ ülich Aachen Research Alliance (JARA)-Fundamentals of Future Information Technology, 52425 Jülich, Germany \\ ${ }^{3}$ Institut für Festkörpertheorie, Westfälische Wilhelms-Universität Münster, 48149 Münster, Germany
}

(Received 26 February 2015; published 6 July 2015)

\begin{abstract}
We introduce a scanning probe technique that enables three-dimensional imaging of local electrostatic potential fields with subnanometer resolution. Registering single electron charging events of a molecular quantum dot attached to the tip of an atomic force microscope operated at $5 \mathrm{~K}$, equipped with a qPlus tuning fork, we image the quadrupole field of a single molecule. To demonstrate quantitative measurements, we investigate the dipole field of a single metal adatom adsorbed on a metal surface. We show that because of its high sensitivity the technique can probe electrostatic potentials at large distances from their sources, which should allow for the imaging of samples with increased surface roughness.
\end{abstract}

DOI: 10.1103/PhysRevLett.115.026101

The atomic structure of matter inevitably leads to local electrostatic fields in the vicinity of nanoscale objects even if they are neutral [1]. Hence, electrostatic forces often dominate the interactions between nanostructures, but experimental access to such local electrostatic fields is a challenge, Kelvin probe force microscopy (KPFM) being the most promising attempt to address it so far [2-4]. However, since KPFM measures the contact potential difference between surfaces, which by definition are extended objects, it inevitably involves lateral averaging, especially for larger probe-to-surface distances. True threedimensional imaging of local electrostatic fields in a broad distance range is therefore difficult with KPFM [5].

Here we introduce a scanning probe technique, scanning quantum dot microscopy (SQDM), that provides a contactfree measurement of the electrostatic potential in all three spatial dimensions, without the drawback of distancedependent averaging. This is possible because SQDM, unlike KPFM, directly probes the local electrostatic potential at a well-defined subnanometer-sized spot in the junction. SQDM also shows a remarkable sensitivity that allows, e.g., the detection of the electrostatic potential $\sim 6 \mathrm{~nm}$ above a single adatom on a metal surface.

We start by describing the general working principle of SQDM, independent of the specific nature of the employed quantum dot (QD). We image the electrostatic potential using a nanometer-sized QD attached to the apex of a scanning probe tip [Fig. 1(a)]. In the experiment, the electronic levels of the QD are gated with respect to the

Published by the American Physical Society under the terms of the Creative Commons Attribution 3.0 License. Further distribution of this work must maintain attribution to the author(s) and the published article's title, journal citation, and DOI.
PACS numbers: 68.37.-d, 73.23.Hk, 82.37.Gk, 85.65.+h

Fermi level $E_{\mathrm{F}}$ of the tip by applying a bias voltage to the tip-surface junction [Fig. 1(b)] [6-10]. In this way, the charge state of the QD can be changed, e.g., if the bias voltage $V$ applied to the junction reaches a critical value $V^{-}$ that aligns one of the QD's occupied electronic levels with $E_{\mathrm{F}}$, this level is depopulated [Fig. 1(b)]. With this device, the measurement of a local electrostatic potential field (a)

$\mathrm{N}$
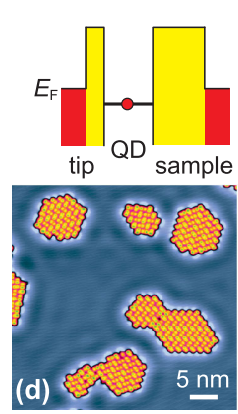

(b)
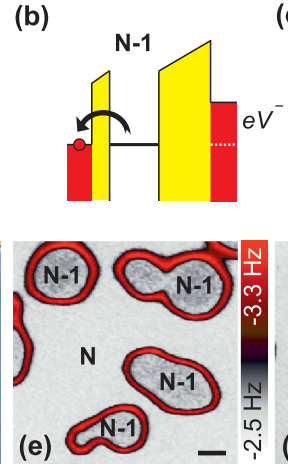

(c)
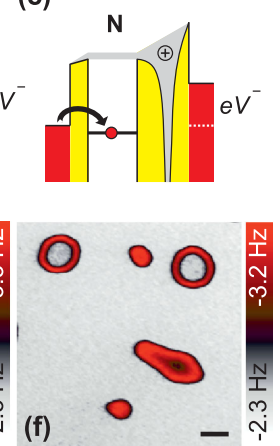

FIG. 1 (color). Working principle of SQDM. (a)-(c) Energy diagrams showing the QD attached to a scanning probe tip. (a) In the absence of a sample bias, a given level of the QD is occupied (QD charge state $N$ ). (b) When a critical sample bias $V^{-}$is reached, one electron tunnels from the QD into the tip (QD charge state $N-1$ ). (c) If a local charge at the surface modifies the potential in the junction, the QD level shifts and becomes reoccupied (QD charge state $N$ ). (d) STM image of monolayer PTCDA islands on $\operatorname{Ag}(111)$. Here, and on all further images, a $5 \mathrm{~nm}$ scale bar is shown. (e) Const. height $\Delta f$ image of the area in (d) recorded at $z_{\text {tip }}=3 \mathrm{~nm}$ [for definition, see Fig. 2(a)] and $V=-990 \mathrm{mV}$. Prominently visible in red are lines where the QD changes its occupation between $N$ and $N-1$. The charge states of the QD in the different regions are labeled. (f) Same as (e), but recorded at $V=-910 \mathrm{mV}$. 
$\Phi(x, y, z)$, caused for example by a surface adsorbate, is possible because the electronic levels of the QD shift in response to any perturbation of the potential at the position $(x, y, z)$ of the QD. These shifts can be detected by their effect on the charge state [Fig. 1(c)]. Detecting charging events of the QD while scanning the three-dimensional half-space above the surface is the working principle of our method.

Figures 1(e) and 1(f), recorded $3 \mathrm{~nm}$ above islands of perylene tetracarboxylic dianhydride (PTCDA) on the $\operatorname{Ag}(111)$ surface, provide an initial illustration of SQDM imaging. The red contours in Figs. 1(e) and 1(f) mark locations where the QD changes its charge state. Note that these contours follow the shape of the standing wave pattern [Fig. 1(d)], which is formed by the surface state as it is scattered by the perturbed electrostatic potential in the surface [11]. This is an indication that the QD is indeed sensitive to the electrostatic potential created by the sample. In the remainder of the Letter, we present experimental results that unambiguously confirm this conjecture.

We now turn to a description of the QD used in the present work. Since the spatial resolution of SQDM is related to the size of the QD, we chose a single molecule as the QD to optimize it. Two questions then arise: is it possible to controllably attach a single molecule to the tip of the scanning probe microscope, and does this molecule indeed exhibit the properties of a QD?

Regarding the first question, we were able to attach both single PTCDA and naphthalene tetracarboxylic dianhydride (NTCDA) molecules [Figs. 2(a) and 2(b)] to the apex of the tip of a CREATEC qPlus tuning fork [12] noncontact atomic force/scanning tunneling microscope (NC-AFM/ STM), operated at $5 \mathrm{~K}$ and in ultrahigh vacuum. This is achieved with a well-documented manipulation routine [13-16]: an isolated PTCDA or NTCDA molecule, adsorbed on $\operatorname{Ag}(111)$, is approached by the silver-terminated AFM/STM tip directly above one of its corner oxygen atoms. At a tip-surface distance of $\sim 6.5 \AA$, the chosen oxygen atom flips up by $\sim 1.4 \AA$ to establish a chemical bond to the apex of the metal tip [13]. By this oxygen-silver bond, the entire molecule can be lifted off the surface $[13,14]$. As the final bond between the molecule and the surface is broken, the attractive interaction with the surface aligns the molecule perpendicularly to the surface $[15,16]$ in a configuration in which the molecule is hanging from the tip by its oxygen-silver bond [Fig. 2(a)] [17].

The tip-suspended PTCDA and NTCDA molecules behave as QDs because their frontier orbitals have $\pi$ character and a weak amplitude at the corner oxygen atoms. Moreover, in the given configuration, the lobes point in a direction that minimizes overlap with the tip; hence, the bond to the tip acts as an insulating spacer, which prevents their hybridization with the tip. This results in very sharp line widths $[13,18]$ and, equally importantly, in the possibility to gate these levels because a sizeable fraction of

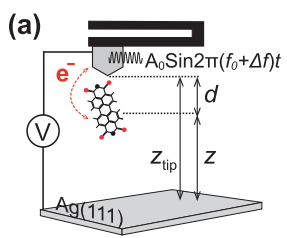

(b)
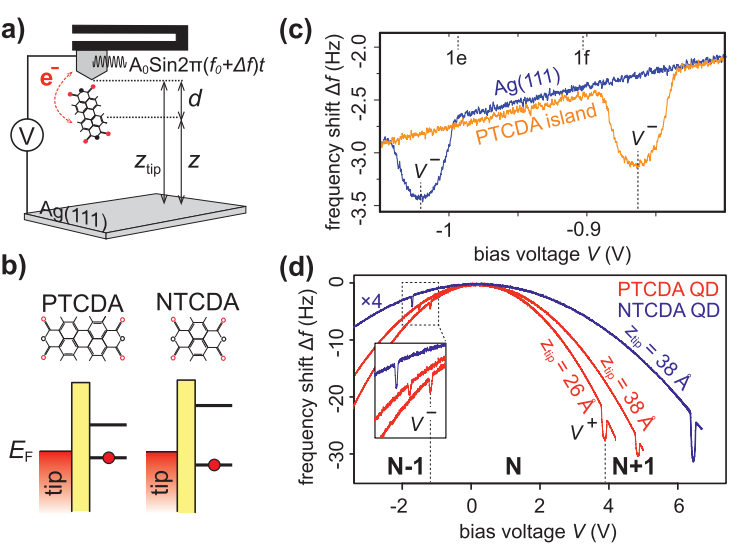

FIG. 2 (color). SQDM with a molecular QD and a NC-AFM. (a) Schematic view of the QD sensor: A single PTCDA molecule is chemically bonded to the AFM tip via a corner oxygen atom. The definitions of $z_{\text {tip }}, z$, and $d$ are indicated. The calibration of $z_{\text {tip }}$ was performed as described in Ref. [16]. (b) Schematics of the energy level alignment of the PTCDA and NTCDA QD tips. (c) $\Delta f(V)$ spectra taken with the PTCDA QD tip at $z_{\text {tip }}=3 \mathrm{~nm}$ above the clean $\operatorname{Ag}(111)$ surface (blue) or above a PTCDA island (orange). The center of each dip determines $V^{-}$. The voltages used for scanning Figs. 1(e) and 1(f) are indicated at the top. (d) $\Delta f(V)$ spectra, recorded with PTCDA (red) and NTCDA (blue) QD tips above the bare $\operatorname{Ag}(111)$ surface. The NTCDA spectrum is multiplied by 4 . For the PTCDA spectrum, recorded at $z_{\text {tip }}=26 \AA$, the QD charge states $N-1, N$, and $N+1$ are indicated.

the applied bias voltage drops over the insulating oxygentip bond. It should be noted, however, that in some respects the properties of our molecular QDs differ from those of more conventional QDs: first, due to large (and sizedependent) intramolecular Coulomb repulsion, the charging energies are in the $\mathrm{eV}$ rather than the $\mathrm{meV}$ range, and second, particle-hole symmetry is broken [19]. In fact, while both NTCDA and PTCDA show the same functionality, the actual level alignment and smaller level spacing in PTCDA [Fig. 2(b)] make it more convenient to work with. Therefore, we focus on experiments with the PTCDA QD tip. The data obtained with NTCDA can be found in the Supplemental Material [18].

Since electrostatic potential measurements in SQDM are based on changes of the QD's electron occupation, a sensitive detection of charging events is crucial. Here this is accomplished by registering abrupt steps in the tipsample force that accompany the change of the QD's charge state $[6-8,10]$. In the qPlus NC-AFM, these steps show up as sharp dips in the frequency shift curve $\Delta f(V)$ [Fig. 2(c)] [12,20,21].

Based on our detection method, SQDM images can be recorded either by mapping $\Delta f$ [Figs. $1(\mathrm{e})$ and 1(f)] or by plotting constant-height maps of charging voltages $V^{-}$ [Fig. 2(c)]. If only one $\Delta f$ feature is used, the resulting SQDM contrast contains a combination of electrostatic and topography effects. Fortunately, we are able to detect two 
$\Delta f(V)$ features (at $V^{-}$and $V^{+}$), corresponding to the $N \rightarrow$ $N-1$ and $N \rightarrow N+1$ transitions of the QD [Fig. 2(d)] [22]. The simultaneous analysis of both $\Delta f(V)$ features allows for a straightforward disentanglement of topography and electrostatics.

The fact that topographic signatures in the surface can change the charge state of the QD if it is scanned at constant height (i.e., fixed $z$ ) across the surface is naturally explained by changes of the junction capacitance with the distance between tip and sample [7]. The effect is illustrated in Fig. 2(d) by the observation that the absolute values $\left|V^{+}\right|$ and $\left|V^{-}\right|$increase with $z_{\text {tip }}$. We describe this behavior in terms of a "gating efficiency" $\alpha$. A smaller value of $\alpha$ implies that a larger bias is needed to align any given QD level with $E_{\mathrm{F}}$. In contrast to the topography, a local electrostatic potential $\Phi^{*}$ at the position of the QD shifts $V^{-}$and $V^{+}$rigidly on the voltage axis $\left(\Delta V \equiv V^{+}-V^{-}=\right.$const). For a fixed $z$, the separation of $\Phi^{*}$ from topography can be achieved straightforwardly by writing down the charging conditions $E_{\text {hole }}+\alpha e V^{-}+$ $e \Phi^{*}=0$ and $E_{\mathrm{el}}-\alpha e V^{+}-e \Phi^{*}=0\left(E_{\mathrm{el}}\right.$ and $E_{\text {hole }}$ are the electron and hole charging energies of the QD) for transitions $N \rightarrow N-1$ and $N \rightarrow N+1$, respectively, and solving for $\alpha$ and $\Phi^{*}$. The result is $\alpha=C / \Delta V$ and $\Phi^{*}=-C V^{-} / \Delta V+\Phi_{0}^{*}$, where $C$ and $\Phi_{0}^{*}$ are constants that can be determined by a calibration experiment where $\Phi^{*}=$ 0 (see below). Details can be found in the Supplemental Material [18]. The fact that $\Delta V$ is inversely proportional to $\alpha$ shows that it primarily carries information about the topography [23].

We now demonstrate the power of SQDM by mapping out the local electrostatic potential field of a nanostructure. As the latter, we choose a single PTCDA molecule on $\mathrm{Ag}(111)$. Its field is expected to contain two major contributions, a quadrupolar field, produced by the internal charge distribution of the molecule [Fig. 3(a)], and a dipolar field due to the electron transfer from $\operatorname{Ag}(111)$ to PTCDA upon adsorption [24].

Using the PTCDA QD, we measure $V^{-}$and $V^{+}$on a grid above the adsorbed PTCDA molecule and plot in Figs. 3(a)-3(f) the experimental quantities $\Delta V(x, y)$, related to the topography, and $V^{-} / \Delta V(x, y)$, proportional to the electrostatic potential (up to a constant offset). Figures 3(d)-3(f) bear a close resemblance to the expected molecular quadrupole field. This is reinforced by a comparison to the results of a microelectrostatic simulation, in which the internal charge distribution of a gas-phase PTCDA, as calculated by density functional theory (DFT), its screening by the metal, and a homogeneous charge transfer from the metal to the molecule have been taken into account. The simulated images in Figs. 3(g)-3(i) were obtained by fitting the experimental data from Figs. 3(d)-3(f) with a two-parameter model, the first parameter being the charge transfer from $\operatorname{Ag}(111)$ to PTCDA $(q=-0.09 e)$, and the second parameter the distance $z$ from the surface at

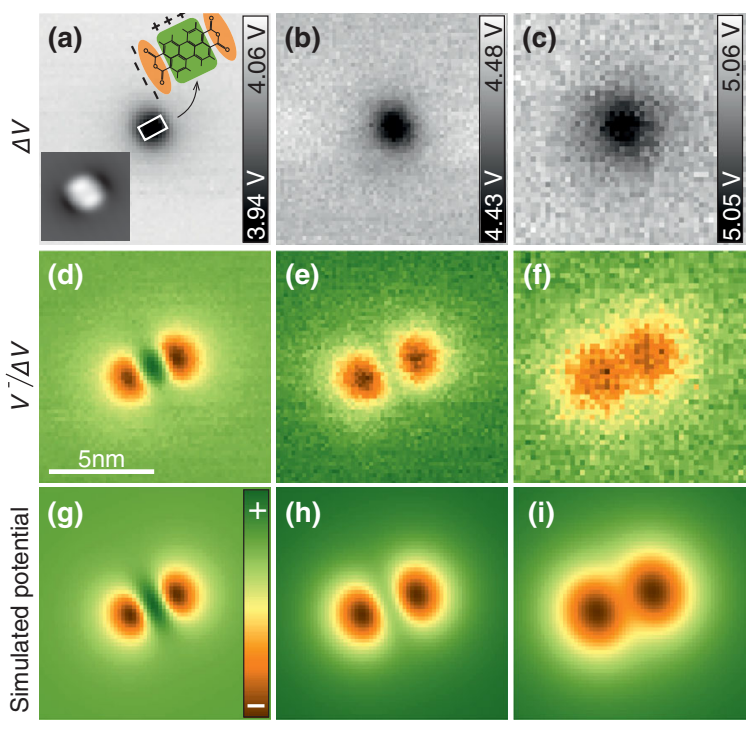

FIG. 3 (color). PTCDA on $\operatorname{Ag}(111)$ : separation of topographic and electrostatic contrasts in SQDM. (a)-(c) Experimental $\Delta V(x, y)$ maps (related to topography, cf. text) recorded with the PTCDA QD tip above an isolated PTCDA molecule adsorbed on the $\operatorname{Ag}(111)$ surface. (a) A white rectangle outlines the size of the PTCDA molecule. In the upper right corner, an enlarged structure formula, on which the quadrupolar charge distribution is indicated, is displayed. The inset in the bottom left corner shows an STM image [scale as in (a)]. (d)-(f) Experimental $V^{-} / \Delta V(x, y)$ maps (related to electrostatic potential, cf. text) of the same area as in (a)-(c). Maps (a) and (d) were recorded at $z_{\text {tip }}=24 \AA$, (b) and (e) at $28 \AA$, and (c) and (f) at $36 \AA$. (g)(i) Simulated electrostatic potential of adsorbed PTCDA at $z=16 \AA(\mathrm{g}), 22 \AA(\mathrm{h})$, and $28 \AA$ (i). The color scales in (d)-(i) were adjusted to optimize the contrast of each figure.

which the simulated potential is plotted [16 $\mathrm{A}, 22 \AA$, and $28 \AA$ in Figs. 3(g), 3(h), and 3(i), respectively]. Remarkably, comparing the obtained distances $z$ with the experimental $z_{\text {tip }}$ [24 $\mathrm{A}, 28 \AA$, and $36 \AA$ in Figs. 3(d), 3(e), and 3(f)], we obtain an estimate of $d=(7 \pm 1) \AA$ in Fig. 2(a). This shows that the electrostatic potential is probed at a point approximately $7 \AA$ below the tip apex, hence at the position of the PTCDA $\mathrm{QD}$, as expected from the proposed junction geometry [Fig. 2(a)].

We now choose the Smoluchowski dipole [1], created here by a single $\mathrm{Ag}$ atom adsorbed on $\operatorname{Ag}(111)$ [Figs. 4(a)-4(c)], to demonstrate that SQDM can deliver quantitative three-dimensional electrostatic potential imaging. To this end, reference measurements $V_{0}^{-}(z)$ and $V_{0}^{+}(z)$ (for a fixed set of heights $z$ ) at locations where the local electrostatic potential $\Phi^{*}$ is taken to be zero, e.g., above bare $\operatorname{Ag}(111)$, can be used to eliminate the $z$-dependent constants $C$ and $\Phi_{0}^{*}$. In this way, $\Phi^{*}$ can be evaluated from $\Phi^{*}(x, y, z)=-\alpha_{0}(z)\left\{\left[V^{-}(x, y, z) / \Delta V(x, y, z)\right] \Delta V_{0}(z)-\right.$ $\left.V_{0}^{-}(z)\right\}$ [18], where $\alpha_{0}(z)$ is the $z$-dependent gating efficiency when the QD tip is above bare $\operatorname{Ag}(111)$. In the simplest case, $\alpha_{0}(z)=d /(z+d)$, if a plate capacitor geometry is assumed. Figure 4(d) shows the experimental 

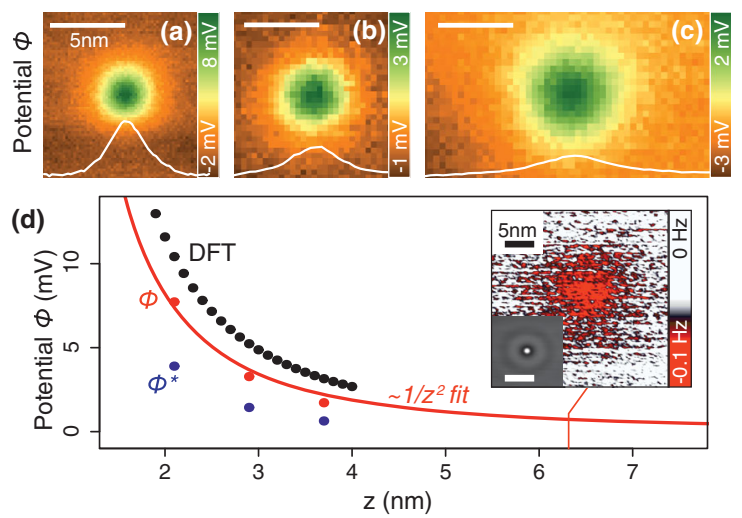

FIG. 4 (color). Smoluchowski dipole of an adatom: quantitative electrostatic potential measurements with SQDM. (a)-(c) Electrostatic potential maps $\Phi(x, y)$ measured above a silver adatom on $\operatorname{Ag}(111)$ at $z=21 \AA$ (a), $29 \AA$ (b), and $37 \AA$ (c). The scale bars show the absolute values of the electrostatic potential $\Phi$, obtained as described in the text. Line profiles through the adatom are shown in white. (d) Comparison of the experimental $\Phi^{\star}$ (blue), $\Phi$ (red), and DFT-calculated (black) potentials vertically above the adatom. The red line shows a $1 /\left(z-z_{0}\right)^{2}$ fit of the experimental data, where $z_{0}$ was obtained by fitting the DFT dataset. For the experimental data, $z$ is the distance between the point inside the QD, at which the electrostatic potential is measured, and the surface. For DFT, $z$ is the distance from the surface at which the potential of the adatom was calculated. (Inset) Constant height raw $\Delta f$ image recorded at $z=6.3 \mathrm{~nm}$ with an applied bias of $V=9.6 \mathrm{~V}$, close to $V^{+}$for this $z$. (Bottom left corner) STM image of the adatom.

electrostatic potential vertically above the adatom, evaluated by the above formula, in comparison to the result of a DFT calculation [25].

Before making the comparison, one should note that DFT yields the electrostatic potential $\Phi$ in the absence of the tip. It is clear that the grounded tip screens the local electrostatic potential $\Phi$ to a smaller value $\Phi^{*}$. Taking into the account this screening [28], we obtain an experimental $\Phi$ that is $\sim 70 \%$ of the DFT values. We consider this agreement a remarkable verification of quantitative electrostatic potential mapping in SQDM. The remaining discrepancy between theory and experiment can be explained by an increase of $\alpha$ (in comparison to the plate capacitor model), caused by the curvature of the metal tip used in the experiment. We note that this influence can be quantified by measuring a structure whose electrostatic potential is known and then transferred to any other experiment with the same tip.

Finally, we comment on the sensitivity of our electrostatic potential field measurement. An accuracy of $\sim 1 \mathrm{mV}$ in measuring $V^{-}, V^{+}$translates into $\Phi^{*}(z)$ from the adatom being detectable at $z$ up to $\sim 4 \mathrm{~nm}$. The inset in Fig. 4(d) shows that, imaging $\Delta f$ directly, the Smoluchowski dipole field of the adatom is observed even at a distance of $6.3 \mathrm{~nm}$ from the surface.

In conclusion, we have reported a scanning probe technique that is able to provide truly three-dimensional, so far elusive, maps of the electrostatic potential field with nanometer resolution. Since the QD serves as a sensor of the electrostatic potential that at the same time transduces this signal to a charging event, the technique is a particularly interesting variant of the general sensor-transducer concept for scanning probe microscopy introduced earlier [29-31]. Here, however, the transduction involves electronic rather than the mechanical degrees of freedom that were utilized in previous work.

As a consequence of its high sensitivity, SQDM may in the future be applied to the characterization of rough and high-aspect-ratio samples such as semiconductor devices or large biomolecular structures [18]. Moreover, the combination of high sensitivity and spatial resolution suggests the possibility of reading nanoscale electric memory cells entirely contact and current free. Finally, we stress that the molecular QD realization of SQDM reported here, although particularly attractive, does not exhaust all possibilities [32]. SQDM probes with nano-fabricated QDs on standard silicon AFM cantilevers may in the future extend the applicability beyond ultrahigh vacuum and cryogenic temperatures.

T. D., P. K., and M.R. gratefully acknowledge the computing time granted by the John von Neumann Institute for Computing (NIC) and provided on the supercomputer JUROPA at Jülich Supercomputing Centre (JSC). R. T. acknowledges discussions with D. Ryndyk (TUDresden) and H. Vázquez (AS CR).

*r.temirov@fz-juelich.de

[1] R. Smoluchowski, Anisotropy of the Electronic Work Function of Metals, Phys. Rev. 60, 661 (1941).

[2] M. Nonnenmacher, M. P. O'Boyle, and H. K. Wickramasinghe, Kelvin probe force microscopy, Appl. Phys. Lett. 58, 2921 (1991).

[3] L. Gross, F. Mohn, P. Liljeroth, J. Repp, F. Giessibl, and G. Meyer, Measuring the charge state of an adatom with noncontact atomic force microscopy, Science 324, 1428 (2009).

[4] F. Mohn, L. Gross, N. Moll, and G. Meyer, Imaging the charge distribution within a single molecule, Nat. Nanotechnol. 7, 227 (2012).

[5] B. Schuler, S. Liu,Y. Geng, S. Decurtins, G. Meyer, and L. Gross, Contrast formation in Kelvin probe force microscopy of single $\pi$-conjugated molecules, Nano Lett. 14, 3342 (2014).

[6] M. T. Woodside and P. L. McEuen, Scanned probe imaging of single-electron charge states in nanotube quantum dots, Science 296, 1098 (2002).

[7] R. Stomp, Y. Miyahara, S. Schaer, Q. Sun, H. Guo, P. Grutter, S. Studenikin, P. Poole, and A. Sachrajda, Detection of Single-Electron Charging in an Individual InAs Quantum Dot by Noncontact Atomic-Force Microscopy, Phys. Rev. Lett. 94, 056802 (2005).

[8] L. Cockins, Y. Miyahara, S. D. Bennett, A. Clerk, S. Studenikin, P. Poole, and P. Grutter, Energy levels of 
few-electron quantum dots imaged and characterized by atomic force microscopy, Proc. Natl. Acad. Sci. U.S.A. 107, 9496 (2010).

[9] I. Fernandez-Torrente, D. Kreikemeyer-Lorenzo, A. Stróżecka, K. J. Franke, and J. I. Pascual, Gating the Charge State of Single Molecules by Local Electric Fields, Phys. Rev. Lett. 108, 036801 (2012).

[10] C. Lotze, PhD Thesis, Freie Universität Berlin, 2013.

[11] M. F. Crommie, C. P. Lutz, and D. M. Eigler, Imaging standing waves in a two-dimensional electron gas, Nature (London) 363, 524 (1993).

[12] F. J. Giessibl, Advances in atomic force microscopy, Rev. Mod. Phys. 75, 949 (2003).

[13] C. Toher, R. Temirov, A. Greuling, F. Pump, M. Kaczmarski, G. Cuniberti, and F. S. Tautz, Electrical transport through a mechanically gated molecular wire, Phys. Rev. B, 83, 155402 (2011).

[14] N. Fournier, C. Wagner, C. Weiss, R. Temirov, and F. S. Tautz, Force-controlled lifting of molecular wires, Phys. Rev. B 84, 035435 (2011).

[15] C. Wagner, N. Fournier, F. S. Tautz, and R. Temirov, Measurement of the Binding Energies of the Organic-Metal Perylene-Tetracarboxylic-Dianhydride/Au(111) Bonds by Molecular Manipulation using an Atomic Force Microscope, Phys. Rev. Lett. 109, 076102 (2012).

[16] C. Wagner, N. Fournier, V. G. Ruiz, C. Li, K. Müllen, M. Rohlfing, A. Tkatchenko, R. Temirov, and F.S. Tautz, Non-additivity of molecule-surface van der Waals potentials from force measurements, Nat. Commun. 5, 5568 (2014).

[17] This configuration has been validated, e.g., by DFT simulations [13] and by quantitative measurements of the van der Waals attraction in this geometry [16], and it is in fact further supported by the QD properties of the tip-suspended molecules because any overlap of the molecular $\pi$ electron system with the metal tip would destroy these properties.

[18] See Supplemental Material at http://link.aps.org/ supplemental/10.1103/PhysRevLett.115.026101 for a PTCDA QD level width measurement, the derivation of the expressions for $\alpha$ and $\Phi^{*}$, and for additional SQDM images recorded with PTCDA and NTCDA QDs.

[19] We explain the breaking of particle-hole symmetry, i.e., the fact that unlike in Ref. [7] $V^{-}$and $V^{+}$are not symmetric with respect to zero, by the presence of electric fields that originate from charge transfer effects that are caused by the attachment of the molecule to the tip. Our data suggest that in the state $N$ the molecules are slightly negatively charged. Hence, it requires less voltage to decrease the occupation of the dot $\left(V^{-}\right)$than to increase it $\left(V^{+}\right)$.

[20] Our NC AFM, operated in the frequency modulation mode, measures the gradient $d F_{z} / d z$ of the vertical force $F_{z}$ acting on the tip, which oscillates with sub- $\AA$ amplitudes [12].

[21] We note that the molecular QDs employed here show no dissipation signal at these charging events because, due to the meV level widths, the corresponding time scales are of the order of picoseconds and thus much shorter than the oscillation period of the qPlus sensor $(\sim 30 \mu \mathrm{s})$. Hence, no charging hysteresis and no dissipation will occur.

[22] Note that $V^{-}$and $V^{+}$charging events appear on top of the parabola, which originates from the attractive interaction between the opposing electrodes of the biased tip-surface junction [3].

[23] Note that local variations of the surface dielectric constant can also change the gating efficiency $\alpha$.

[24] Y. Zou, L. Kilian, A. Schöll, T. Schmidt, R. Fink, and E. Umbach, Chemical bonding of PTCDA on Ag surfaces and the formation of interface states, Surf. Sci. 600, 1240 (2006).

[25] The DFT calculation was carried out using the SIESTA package $[26,27]$. The exchange-correlation part was treated within the local-density approximation, which gives realistic results for strongly bound adsorbates like the Ag adatom considered here. The adatom adsorbs at the hollow site with a height of $2.11 \AA$, which is $0.25 \AA$ closer to the surface than its ideal position would be. In order to calculate the electrostatic potential in real space, we decompose the charge density into two parts, by the clean surface and by the adatom. The main contribution of the $\mathrm{Ag}$ adatom originates from a region of about $4 \AA$ around the adatom. Since the potential of the clean neutral surface decays exponentially with increasing height, only the charge density contribution due to the adatom causes a measurable electrostatic potential at the position of the QD. This potential is evaluated from the charge density by solving Poisson's equation in real space, without any supercell periodicity.

[26] P. Ordejon, E. Artacho, and J. M. Soler, Self-consistent Order-N density functional calculations for very large systems, Phys. Rev. B 53, R10441 (1996).

[27] J. M. Soler, E. Artacho, J. D. Gale, A. Garcia, J. Junquera, P. Ordejón, and D. Sánchez-Portal, The SIESTA method for $a b$ initio order-N materials simulation, J. Phys. Condens. Matter 14, 2745 (2002).

[28] The screening was evaluated by considering an elementary electrostatic model of a point charge in front of an infinite metal surface.

[29] G. Kichin, C. Weiss, C. Wagner, F. S. Tautz, and R. Temirov, Single molecule and single atom sensors for atomic resolution imaging of chemically complex surfaces, J. Am. Chem. Soc. 133, 16847 (2011).

[30] P. Hapala, G. Kichin, C. Wagner, F. S. Tautz, and P. Jelínek, Mechanism of high-resolution STM/AFM imaging with functionalized tips, Phys. Rev. B 90, 085421 (2014).

[31] C. Wagner and R. Temirov, Tunnelling junctions with additional degrees of freedom: An extended toolbox of scanning probe microscopy, Prog. Surf. Sci. 90, 194 (2015).

[32] M. Hauser, J. Smoliner, C. Eder, G. Ploner, G. Strasser, and E. Gornik, Single quantum dots as scanning tunneling microscope tips, Superlattices Microstruct. 20, 623 (1996). 